\title{
ACUTE PANCREATITIS IN PREGNANCY: A CASE REPORT
}

\author{
K. Saritha ${ }^{1}$, V. Prathyusha ${ }^{2}$
}

\section{HOW TO CITE THIS ARTICLE:}

K. Saritha, V. Prathyusha. "Acute Pancreatitis in Pregnancy: A Case Report". Journal of Evolution of Medical and Dental Sciences 2015; Vol. 4, Issue 13, February 12; Page: 2228-2230, D0I: 10.14260/jemds/2015/321

ABSTRACT: Acute pancreatitis in pregnancy is rarely seen. It can be associated with maternal mortality and foetal loss. Prompt diagnosis and treatment is essential. We report a case of Primigravida with 36 weeks gestation with established preterm labour with severe epigastric pain. Diagnosis and management modalities are discussed.

KEYWORDS: Acute pancreatitis, Epigastric pain, Pregnancy.

INTRODUCTION: Acute pancreatitis in pregnancy is rare and occurs infrequently and has a reported incidence of approximately 1 in 1000 to 3 in 10000 births. ${ }^{1,2}$ Recently, Zhang et al reported the incidence of pregnancy associated acute pancreatitis to be $2.7 \% .^{3}$ Most common aetiology is biliary (Gall stone or biliary sludge). Other important causes are hyperlipidaemia and alcohol abuse. It is difficult to diagnose and delay in diagnosis and treatment results threat to maternal and foetal health. Older studies reported high perinatal mortality due to premature delivery. ${ }^{4}$ Recent advances in diagnostic imaging and availability of neonatal intensive care facilities has resulted in improved maternal and neonatal outcome. We report a case of acute pancreatitis in labour and discuss its aetiology, diagnosis and management.

CASE REPORT: A 20 years old Primigravida was referred from the District hospital at 8 p.m. with history of 36 weeks of gestation with complaints of severe upper abdominal pain and vomiting of one day duration. The pain was sudden in onset, mainly in the epigastric region, radiating to the back. Vomiting one episode was non projectile and non-bilious in nature. There was no associated headache, blurring of vision, oedema of feet, face, or abdominal wall, jaundice, haematemesis, decreased urine output. There was no vaginal bleeding.

On admission Temperature was normal, pulse rate 108/minute, blood pressure 110/70 m.m. $\mathrm{Hg}$, and respiratory rate $22 /$ minute. Cardiovascular, respiratory systems were normal. There was tenderness in the epigastric region but no abdominal guarding or rigidity.

Obstetric examination revealed a uterus of 36 weeks gestation, mildly acting with foetus in longitudinal lie and cephalic presentation, head engaged and liquor adequate. Foetal heart rate was 144 beats per minute and regular. Uterus was not tense or tender and the foetal parts were easily palpable between contractions.

Per vaginal examination showed cervix $80 \%$ effaced, os $3 \mathrm{~cm}$, membranes present. The presenting part was at -2 station, could be pushed to -1 station. Pelvis was gynaecoid and adequate for vaginal delivery.

Routine investigations were done. Haemoglobin $11.8 \mathrm{gm} / \mathrm{dl}$, total leucocyte count 12800 cells /c mm, urine for albumin and sugar negative, random blood sugar was $102 \mathrm{mg} \% \mathrm{HIV}$, HBsAg were nonreactive. Electrocardiogram was normal. Bleeding time and clotting time were normal.

Emergency USG showed a live foetus with estimated foetal weight of $2.3 \mathrm{~kg}$, liquor adequate, and placenta in upper segment of uterus, grade 2, anterior and no retro placental clots were noted.

Blood sample was sent for serum amylase and lactic dehydrogenase. 
She was managed with nil by mouth, intravenous fluids, and injections of pantoprazole, hyoscine bromide and tramadol. Meanwhile her labour progressed and a live $2.5 \mathrm{~kg}$ baby with good Apgar score was delivered by outlet forceps 6 hours after admission. Baby was sent to the neonatal intensive care unit in view of borderline prematurity.

But her epigastric pain continued and she developed abdominal distension with sluggish bowel sounds. Ryles tube aspiration done. A repeat USG of abdomen and pelvis showed uterus postpartum status, pancreas bulky in size with hypo echoic texture, minimal perihepatic free fluid, bilateral minimal pleural effusions. Liver, gall bladder, kidneys were normal. Gall bladder was empty.

A contrast enhanced CT scan was advised.

Her serum amylase level was elevated 1684 IU (normal 28-100), lactic dehydrogenase levels were also elevated 278 IU (normal 62-155 IU). Diagnosis of acute pancreatitis was made and she was shifted to the Gastroenterology department.

CT scan of abdomen showed mild hepatomegaly, bulky pancreas with peripancreatic fatty strands, bulky uterus (postpartum status), mild ascites and bilateral pleural effusion with basal atelectasis. 2D Echo showed normal study.

With conservative management she improved and was discharged from hospital after one week. Follow up after 15 days was normal.

DISCUSSION: Acute pancreatitis complicating pregnancy is a rare and challenging problem. Most important aetiological factors are gall stone disease and hypertriglyceridemia. Pregnancy increases the level of serum cholesterol, causes biliary stasis and induces gall stone formation. ${ }^{4}$ Plasma triglycerides increase to 2-4 times in pregnancy, principally in the third trimester, due to increased triglyceride-rich lipoprotein production and decreased lipoprotein lipase activity. ${ }^{5,6}$ Other aetiological factors for acute pancreatitis include alcohol abuse, diabetes mellitus, and rarely, hyperparathyroidism, connective tissue diseases, abdominal surgery, infections or iatrogenic causes such as diuretics, antibiotics and antihypertensive drugs. ${ }^{4}$

The most common misdiagnosis of pancreatitis in the first trimester is hyperemesis gravidarum. In women presenting with severe nausea and vomiting in the first trimester consider obtaining amylase, lipase levels and liver function tests, which when elevated are diagnostic for pancreatitis. $^{7}$ The differential diagnosis in a pregnant woman with upper abdominal pain and vomiting in the later weeks of gestation are placental abruption, severe preeclampsia or gastritis. ${ }^{8}$

These conditions were ruled out because uterus was not tense, non-tender, no bleeding per vagina, blood pressure was normal, no protein in the urine, no relief with antacids.

Diagnosis of acute pancreatitis is supported by increased serum amylase and lipase as well as triglyceride levels, calcium levels and a complete blood count. Serum amylase level is usually considered as a reliable marker of acute pancreatitis in pregnant women. However, patients with necrotic pancreatitis or hypertriglyceridemia induced acute pancreatitis may have normal serum levels of amylase and this result can be interpreted as not having acute pancreatitis in pregnancy. In addition to above tests certain imaging methods are necessary. ${ }^{9}$

Although abdominal ultrasound is an ideal technique to diagnose acute pancreatitis in pregnancy, enlarged gravid uterus and accompanying ileus make pancreatic shadow invisible. Computed tomography (CT), endoscopic ultrasound and magnetic resonance cholangiopancreato- 
graphy (MRCP) are available for diagnosing a biliary aetiology for acute pancreatitis. The usefulness of CT is substantially restricted in pregnancy because of the potential radiation exposure to foetus. ${ }^{4}$

In conclusion, acute pancreatitis pregnancy complicating is a rare condition. Prompt diagnosis and management will prevent complications such as sepsis, perforation and multiorgan dysfunction. The goal of the treatment in acute pancreatitis in pregnancy is to maintain both maternal and foetal well-being.

\section{REFERENCES:}

1. Pitchumoni CS, Yegneswaran B. Acute pancreatitis in pregnancy World J Gastroenterol 2009; 15: 5646

2. Qihui C, Xiping Z, Xianfeng D. Clinical study on acute pancreatitis in pregnancy in 26 cases Gastroenterol Res Pract 2012; 2012: 2719-25.

3. Zhang DL, Huang Y, Yan L, Phu A, Ran X, Li SS. Thirty eight cases of acute pancreatitis in pregnancy: a 6-year single center retrospective analysis. J Huazhong Univ Sci Technolog Med Sci 2013; 33: 361-367.

4. Ji Yeon Kim, Sung Hoon Jung, Hyung Wook Choi, Dong Jin Song, Chel Yoon Jeong et al. Acute idiopathic pancreatitis in pregnancy: A case study World J Gastroenterol 2014;20(43): 1636416367.

5. Neill AM, Hackett GA, Overton C, Byrne CD. Active management of acute hyperlipidaemic pancreatitis in pregnancy. J Obstet Gynecol 1998; 18:174-175.

6. Eddy JJ, Gideonsen MD, Song JY, Grobman WA, O'Halloran P. Pancreatitis in pregnancy. Obstet Gynecol 2008; 112: 1075-1081.

7. Sunil Kumar Juneja, Shweta Gupta, Satpal Sing Virk, Pooja Tandon and Vidushi Binda. Acute pancreatitis in pregnancy: A treatment paradigm based on our hospital experience Int J Appl Basic Med Res 2013; 3(2): 122-125.

8. $\mathrm{P}$ Kushtagi, $\mathrm{P}$ Adiga, $\mathrm{S}$ Benjamin, $\mathrm{P}$ Bhat. Acute pancreatitis in the pregnant The Internet Journal of Gynecology and Obstetrics 2009; Vol 12No 2.

9. Ayse Filiz Avsar, Melahat Yildirim and Aysegul Cinkaya. Unexpected fetal demise despite the reactive nonstress test during the conservative management of acute pancreatitis in pregnancy Int J Surg Case Rep 2014; 5 (12): 1047-1049.

\section{AUTHORS:}

1. K. Saritha

2. V. Prathyusha

\section{PARTICULARS OF CONTRIBUTORS:}

1. Associate Professor, Department of Gynaecology, Siddhartha Medial College, Vijayawada, A. P.

2. Post Graduate, Department of Gynaecology, Siddhartha Medial College, Vijayawada, A. P.

FINANCIAL OR OTHER COMPETING INTERESTS: None

\section{NAME ADDRESS EMAIL ID OF THE} CORRESPONDING AUTHOR:

Dr. K. Saritha,

Plot No. 15,

Indian Airlines Colony,

Opposite Football Grounds,

Trimulghrry, Secunderabad-500015.

E-mail: saritha.kottu@gmail.com

Date of Submission: 19/01/2015.

Date of Peer Review: 20/01/2015.

Date of Acceptance: 04/02/2015.

Date of Publishing: 12/02/2015. 